\title{
Implications of Heavy-Quark Spin Symmetry on Heavy-Meson Hadronic Molecules
}

\author{
Feng-Kun Guo, ${ }^{1, *}$ Christoph Hanhart, ${ }^{1,2, \dagger}$ and Ulf-G. Meißner ${ }^{1,2,3, \neq}$ \\ ${ }^{1}$ Institut für Kernphysik and Jülich Center for Hadron Physics, Forschungszentrum Jülich, D-52425 Jülich, Germany \\ ${ }^{2}$ Institute for Advanced Simulation, Forschungszentrum Jülich, D-52425 Jülich, Germany \\ ${ }^{3}$ Helmholtz-Institut für Strahlen- und Kernphysik and Bethe Center for Theoretical Physics, Universität Bonn, \\ D-53115 Bonn, Germany
}

(Received 23 April 2009; published 19 June 2009)

\begin{abstract}
In recent years, many heavy mesons and charmonia were observed which do not fit in the conventional quark model expectations. Some of them are proposed to be hadronic molecules. Here we investigate the consequences of heavy-quark spin symmetry on these heavy-meson hadronic molecules. Heavy-quark spin symmetry enables us to predict new heavy-meson molecules and provides us with a method to test heavy-meson molecule assumptions of some newly observed states. In particular, we predict an $\eta_{c}^{\prime} f_{0}(980)$ bound state as the spin-doublet partner of the $Y(4660)$ proposed as a $\psi^{\prime} f_{0}(980)$ bound state with a mass of $4616_{-6}^{+5} \mathrm{MeV}$ and the prominent decay mode $\eta_{c}^{\prime} \pi \pi$. The width is predicted to be $\Gamma\left(\eta_{c}^{\prime} \pi \pi\right)=60 \pm$ $30 \mathrm{MeV}$. The $\pi^{+} \pi^{-}$invariant mass spectrum and the line shape are calculated. We suggest searching for this state in $B^{ \pm} \rightarrow \eta_{c}^{\prime} K^{ \pm} \pi^{+} \pi^{-}$, whose branching fraction is expected to be large.
\end{abstract}

The chromomagnetic interaction of a heavy quark with gluons is proportional to the magnetic moment of the heavy quark, and is suppressed by the heavy-quark mass (for reviews of heavy-quark symmetry, see Refs. [1,2]). Therefore in the heavy-quark limit $m_{Q} \rightarrow \infty$, the interaction is spin independent, and a new symmetry appears called heavy-quark spin symmetry. Because of this symmetry, there are spin multiplets of both heavy mesons and heavy quarkonia, as, e.g., the $\left\{D, D^{*}\right\}$ and $\left\{\eta_{c}, J / \psi\right\}$. The masses of the members within the same spin multiplet would be degenerate in the heavy-quark limit. In this Letter, we extend this symmetry to possible heavy-meson molecules, which were observed in recent years (for reviews, see, e.g., Refs. [3-6]). By heavy-meson molecules, we mean bound states consisting of a heavy meson or heavy quarkonium and a light hadron, or two heavy mesons. In this Letter, we will focus on the former type.

As an example, let us focus on the $D_{s 0}^{*}(2317)$ and the $D_{s 1}(2460)$ first. They were proposed to be $S$-wave hadronic molecules whose components are mainly $D K$ and $D^{*} K$, respectively [7-12] (for the latest development on the $D_{s 0}^{*}(2317)$ in the hadronic molecular picture, we refer to Refs. [13-16]). Their masses are measured to be [17]

$$
\begin{aligned}
& M_{D_{s 0}^{*}(2317)}=2317.8 \pm 0.6 \mathrm{MeV}, \\
& M_{D_{s 1}(2460)}=2459.6 \pm 0.6 \mathrm{MeV} .
\end{aligned}
$$

Were they the bound states of $D K$ and $D^{*} K$, respectively, the binding energies are

$$
\begin{aligned}
& \epsilon_{D_{s 0}^{*}(2317)}=M_{D}+M_{K}-M_{D_{s 0}^{*}(2317)}=45 \mathrm{MeV}, \\
& \epsilon_{D_{s 1}(2460)}=M_{D^{*}}+M_{K}-M_{D_{s 1}(2460)}=45 \mathrm{MeV},
\end{aligned}
$$

where we have taken the averaged masses within the same isospin multiplets of $D, D^{*}$, and $K$. One notices that the binding energies are the same. For molecular states this appears to be natural: first of all the leading interactions of light mesons with $D$ and $D^{*}$ mesons are independent of the heavy-quark spin and secondly the lightmeson- $D^{(*)}$-meson Greens functions, which provide an important input to the bound-state equations, are to a very good approximation mass-independent as long as evaluated close to the corresponding threshold. As a result, hadronic molecules also fall in spin multiplets, and, most importantly here, the splitting within one multiplet remains the same as the hyperfine splitting between the heavy mesons which are the components of the hadronic molecule.

In the same way the hyperfine splitting within a heavyquarkonium spin multiplet will also be untouched by the interactions with light mesons. The interaction between a heavy-quarkonium and light hadrons occur mainly through exchanging soft gluons. The leading order heavyquarkonium interaction with a soft gluon field comes from the chromoelectric dipole interaction [18-20] which is spin independent. The chromomagnetic interaction is suppressed by $1 / m_{Q}$ [21]. Since any hadron should be color singlet, the number of the exchanged soft gluons should be at least two. Therefore, the suppression of the spin-dependent interactions between a heavy quarkonium and light hadrons is at least $1 / m_{Q}^{2}$. As a result, a bound state of a heavy quarkonium and light hadrons, called hadro-charmonium in Ref. [22], will have partner (s) whose components are the same light hadrons and the spin-multiplet partner(s) of the same heavy quarkonium. The mass splitting within the molecular spin multiplet will be, to a very good approximation, the same as the heavyquarkonium hyperfine splitting. 
This nice feature enables us to predict new heavy-meson molecules and provides us with a method to test heavymeson molecule assumptions of some newly observed states as illustrated in the following.

The Belle Collaboration [23] observed a resonant structure, called $Y(4660)$, in the $\psi^{\prime} \pi^{+} \pi^{-}$final state using the method of initial state radiation. The line shape of the state was fitted with a $P$-wave Breit-Wigner [24] in the experimental paper, and they got $4664 \pm 12 \mathrm{MeV}$. In Ref. [25], we demonstrated that the experimental data support a $\psi^{\prime} f_{0}(980)$ bound-state hypothesis for the $Y(4660)$. As a result of fitting the mass, which gave $M_{Y}=4665_{-5}^{+3} \mathrm{MeV}$, we could calculate the spectral distribution. If this interpretation of the $Y(4660)$ is indeed correct, heavy-quark spin symmetry implies that there is an $\eta_{c}^{\prime} f_{0}(980)$ bound state, to be called $Y_{\eta}$ in the following. The quantum numbers of such a state are $J^{P}=0^{-}$. The $\eta_{c}^{\prime}$ and $\psi^{\prime}$ lie in the same spin multiplet, and their mass splitting is

$$
\Delta M=M_{\psi^{\prime}}-M_{\eta_{c}^{\prime}}=49 \pm 4 \mathrm{MeV} .
$$

From the above analysis, the mass of the $\eta_{c}^{\prime} f_{0}(980)$ bound state would be

$$
M_{Y_{\eta}}=M_{Y(4660)}-\Delta M=4616_{-6}^{+5} \mathrm{MeV},
$$

where the uncertainties of the mass of the $Y(4660)$ and $\Delta M$ were added in quadrature. Similar to the $Y(4660)$ decaying predominantly into $\psi^{\prime} \pi \pi$, the dominant decay channel of the $Y_{\eta}$ would be $Y_{\eta} \rightarrow \eta_{c}^{\prime} \pi \pi$.

If two particles form an $S$-wave bound state which is very close to the threshold, there is a way to modelindependently connect the effective coupling constant of the bound state to its constituents $g$ directly to the molecular admixture of the state [26,27]. Historically, Weinberg used this method to show that the deuteron is not an elementary particle. Especially, one may write for a pure molecule

$$
\frac{g^{2}}{4 \pi}=4\left(m_{1}+m_{2}\right)^{2} \sqrt{\frac{2 \epsilon}{\mu}}\left[1+\mathcal{O}\left(\frac{\sqrt{2 \mu \epsilon}}{\beta}\right)\right],
$$

where $m_{1}$ and $m_{2}$ denote the masses of the constituents, $\epsilon$ the binding energy related to $M$, the mass of the molecule, via $M=m_{1}+m_{2}-\epsilon, \mu=m_{1} m_{2} /\left(m_{1}+m_{2}\right)$ the reduced mass, and $1 / \beta$ the range of the forces.

With the effective coupling constant fixed by Eq. (5), we can predict the $\pi \pi$ invariant mass spectrum and the decay width of the $Y_{\eta} \rightarrow \eta_{c}^{\prime} \pi^{+} \pi^{-}$. Denoting the $\pi^{+} \pi^{-}$invariant mass by $m_{\pi \pi}$, we have for the differential width

$$
\frac{d \Gamma_{Y_{\eta}}}{d m_{\pi \pi}^{2}}=\frac{g^{2} q}{8 \pi M_{Y_{\eta}}^{2}} \rho_{f_{0}}^{\left[\pi^{+} \pi^{-}\right]}\left(m_{\pi \pi}\right),
$$

where $q$ is the magnitude of the three-momentum of the $\eta_{c}^{\prime}$ in the $Y_{\eta}$ rest frame

$$
q=\frac{\sqrt{\left[M_{Y_{\eta}}^{2}-\left(m_{\pi \pi}+M_{\eta_{c}^{\prime}}\right)^{2}\right]\left[M_{Y_{\eta}}^{2}-\left(m_{\pi \pi}-M_{\eta_{c}^{\prime}}\right)^{2}\right]}}{2 M_{Y_{\eta}}},
$$

and $\rho_{f_{0}}^{\left[\pi^{+} \pi^{-}\right]}\left(m_{\pi \pi}\right)$ is the $\pi^{+} \pi^{-}$fraction of the $f_{0}$ spectral function,

$$
\rho_{f_{0}}^{\left[\pi^{+} \pi^{-}\right]}\left(m_{\pi \pi}\right)=\frac{1}{\pi} \frac{\operatorname{Im}\left[\Pi_{f_{0}}^{\pi^{+} \pi^{-}}\left(m_{\pi \pi}\right)\right]}{\left|m_{\pi \pi}^{2}-m_{f_{0}}^{2}+\sum_{a b} \hat{\Pi}_{f_{0}}^{a b}\left(m_{\pi \pi}\right)\right|^{2}},
$$

where $\hat{\Pi}_{f_{0}}^{a b}\left(m_{\pi \pi}\right)=\Pi_{f_{0}}^{a b}\left(m_{\pi \pi}\right)-\operatorname{Re}\left[\Pi_{f_{0}}^{a b}\left(m_{f_{0}}\right)\right]$ denote the renormalized self-energies of the $f_{0}$ with respect to the channel $a b=\pi \pi$ or $K \bar{K}$. Analytic expressions are given in Ref. [28]. The imaginary part of the self-energy of the $f_{0}$ is fixed by unitarity

$$
\operatorname{Im}\left[\Pi_{f_{0}}^{\pi^{+} \pi^{-}}\left(m_{\pi \pi}\right)\right]=m_{f_{0}} \Gamma_{f_{0} \rightarrow \pi^{+} \pi^{-}}\left(m_{\pi \pi}\right),
$$

and

$$
\Gamma_{f_{0} \rightarrow \pi^{+} \pi^{-}}\left(m_{\pi \pi}\right)=\frac{g_{f_{0} \pi^{+} \pi^{-}}^{2}}{16 \pi m_{f_{0}}} \sqrt{1-\frac{4 m_{\pi}^{2}}{m_{\pi \pi}^{2}}} .
$$

The input parameters related to the $f_{0}(980)$ are taken from the fits provided in Ref. [29]. To be specific, we use $m_{f_{0}}=0.9862 \mathrm{GeV}, g_{f_{0} K^{+} K^{-}}=3.87 \mathrm{GeV}$, and $g_{f_{0} \pi^{+} \pi^{-}}=$ $-2.03 \mathrm{GeV}$, which are the central values of the various parameters of fit $K 2$ shown in Table IV of that reference. The couplings for the neutral channels are fixed using the isospin relations. With these parameters, and $M_{\eta_{c}^{\prime}}=$ $3637 \pm 4 \mathrm{MeV}$ [17], the $\pi^{+} \pi^{-}$invariant mass spectrum for the $Y_{\eta}$ decaying into $\eta_{c}^{\prime} \pi^{+} \pi^{-}$is predicted in Fig. 1, where the solid line shows the result using the central values of $M_{\eta_{c}^{\prime}}$ and $M_{Y_{\eta}}$.

Integrating over the $\pi \pi$ invariant mass, and considering both the $\eta_{c}^{\prime} \pi^{+} \pi^{-}$and $\eta_{c}^{\prime} \pi^{0} \pi^{0}$ channels, we get a width of the $Y_{\eta} \rightarrow \eta_{c}^{\prime} \pi \pi$ as $58 \pm 5 \mathrm{MeV}$. Here we only took the leading term of the effective coupling constant as given in Eq. (5), and the uncertainty comes solely from that of the

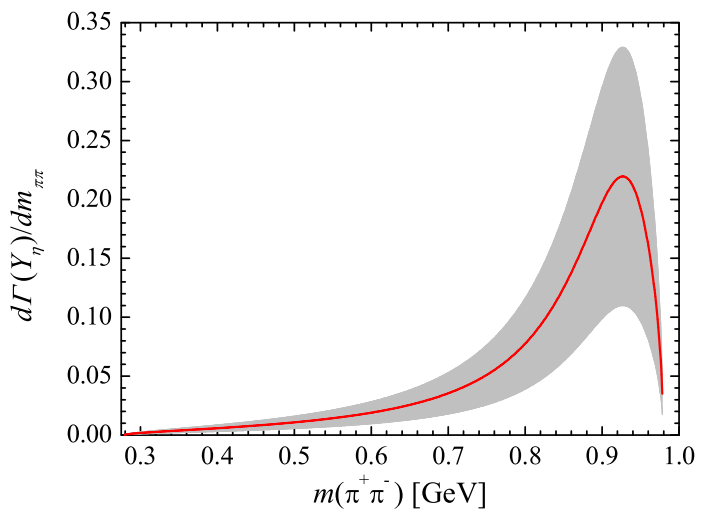

FIG. 1 (color online). The $\pi^{+} \pi^{-}$invariant mass spectrum for the $Y_{\eta} \rightarrow \eta_{c}^{\prime} \pi^{+} \pi^{-}$decay. 
masses of the $Y_{\eta}$ and $\eta_{c}^{\prime}$. However, to get a more realistic estimate of the uncertainty, we need to also estimate, e.g., higher order terms of the effective coupling constant. As stated before, the interactions between a charmonium and light hadrons are mediated by soft gluons, so we may estimate the range of forces as $1 / \beta \approx 1 / \Lambda_{\mathrm{QCD}}$. Noticing $\sqrt{2 \mu \epsilon} \approx 100 \mathrm{MeV}$, the uncertainty of $g^{2}$ from this source, and hence the width, is about $50 \%$. We want to stress that this estimate is clearly quite conservative. Taking the expected inverse mass of the lightest glueball as the range of forces might appear equally justified (and this would lead to a much smaller uncertainty). On the other hand, we do not explicitly include uncertainties from other sources like higher orders in the $1 / m_{Q}$ expansion. Thus, as a result, we get

$$
\Gamma\left(Y_{\eta} \rightarrow \eta_{c}^{\prime} \pi \pi\right)=60 \pm 30 \mathrm{MeV}
$$

and the uncertainty in the $\pi^{+} \pi^{-}$invariant mass spectrum is reflected as the band in Fig. 1. The uncertainty in the coupling constant has a much larger effect on the signal than the one of the mass of the $Y_{\eta}$.

Assuming the width of the $Y_{\eta}$ being saturated by the $\eta_{c}^{\prime} \pi \pi$ final state, the line shape of the $Y_{\eta}$ can also be predicted. For this we use a dispersion integral, which gives us an expression for the $Y$ self-energy, $\Pi_{Y_{\eta}}(M)$, for arbitrary values of $M$

$$
\Pi_{Y_{\eta}}(M)=\frac{1}{\pi} \int_{M_{\mathrm{thr}}^{2}}^{\infty} d s \frac{M_{Y_{\eta}} \Gamma_{Y_{\eta}}^{\mathrm{tot}}(\sqrt{s})}{s-M^{2}-i \epsilon},
$$

where $M_{\text {thr }}=M_{\eta_{c}^{\prime}}+2 m_{\pi}$ denotes the lowest physical threshold of relevance here and $\Gamma_{Y_{\eta}}^{\mathrm{tot}}(\sqrt{s})$ the total width of the $Y_{\eta}$ as a function of $\sqrt{s}$. Note that this treatment is completely consistent with what was done for the $f_{0}$. With the self-energies at hand we may now give the expression

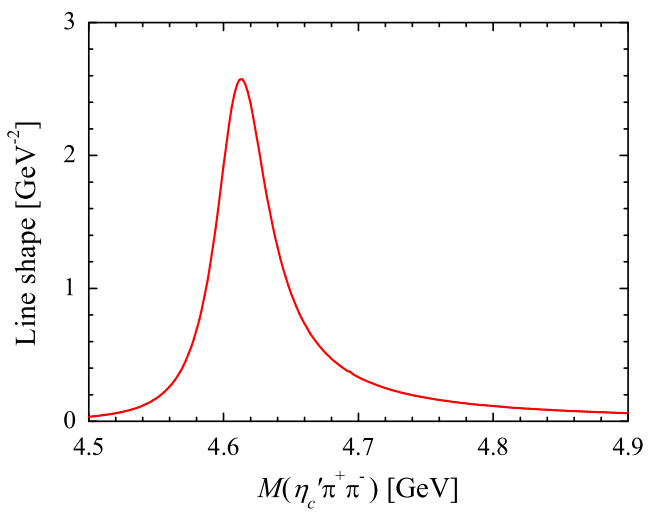

FIG. 2 (color online). Line shape of the $Y_{\eta}$ in the $\eta_{c}^{\prime} \pi^{+} \pi^{-}$ invariant mass distribution. For clarity we only show the line corresponding to the central values of the parameter space. Note that the asymmetry seen in the distribution is an unavoidable consequence of the molecular structure.

for the spectral function of the $Y_{\eta}(4616)$

$$
\rho_{Y_{\eta}}(M)=\frac{M_{Y_{\eta}} \Gamma_{Y_{\eta}}^{\mathrm{tot}}(M)}{\left|M^{2}-M_{Y_{\eta}}^{2}+\hat{\Pi}_{Y_{\eta}}(M)\right|^{2}},
$$

where, as above, we defined $\hat{\Pi}_{Y_{\eta}}(M)=\Pi_{Y_{\eta}}(M)-$ $\operatorname{Re}\left[\Pi_{Y_{\eta}}\left(M_{Y_{\eta}}\right)\right]$. Replacing the total width of the $Y_{\eta}$ in the numerator of Eq. (12) by $\Gamma\left(Y_{\eta} \rightarrow \eta_{c}^{\prime} \pi^{+} \pi^{-}\right)$, one gets $\rho_{Y_{\eta}}^{\left[\eta_{c}^{\prime} \pi^{+} \pi^{-}\right]}(M)$. That is the line shape of the $Y_{\eta}$ in the $\eta_{c}^{\prime} \pi^{+} \pi^{-}$mass distribution as given in Fig. 2.

The proposed $\eta_{c}^{\prime} f_{0}(980)$ bound state can be searched for in $B$ decays. We suggest to search it in $B^{ \pm} \rightarrow \eta_{c}^{\prime} K^{ \pm} \pi^{+} \pi^{-}$. Taking data from Ref. [17] for three measured channels, the branching fraction of the so-far unmeasured decay $B^{ \pm} \rightarrow \eta_{c}^{\prime} K^{ \pm} \pi^{+} \pi^{-}$can be estimated as

$$
\begin{aligned}
\mathcal{B}\left(B^{ \pm} \rightarrow \eta_{c}^{\prime} K^{ \pm} \pi^{+} \pi^{-}\right) & =\mathcal{B}\left(B^{ \pm} \rightarrow \eta_{c}^{\prime} K^{ \pm}\right) \frac{\mathcal{B}\left(B^{ \pm} \rightarrow \psi^{\prime} K^{ \pm} \pi^{+} \pi^{-}\right)}{\mathcal{B}\left(B^{ \pm} \rightarrow \psi^{\prime} K^{ \pm}\right)} \\
& =(3.4 \pm 1.8) \times 10^{-4} \frac{(1.9 \pm 1.2) \times 10^{-3}}{(6.48 \pm 0.35) \times 10^{-4}} \sim 1 \times 10^{-3}
\end{aligned}
$$

Such a large branching fraction offers a great opportunity of finding the $Y_{\eta}$ in the $B$ decays, although we cannot predict $\mathcal{B}\left(B^{ \pm} \rightarrow Y_{\eta} K^{ \pm}\right)$.

In summary, the heavy-quark spin symmetry, which is exact in the heavy-quark limit, is extended to the systems made of a heavy meson or quarkonium and light hadrons. We argue that the hyperfine splitting remains untouched in heavy-meson molecules. Based on this observation, there should be an $\eta_{c}^{\prime} f_{0}(980)$ bound state with a mass of $4616_{-6}^{+5} \mathrm{MeV}$, were the $Y(4660)$ a $\psi^{\prime} f_{0}(980)$ bound state as suggested in Ref. [25]. Such a bound state would decay mainly into $\eta_{c}^{\prime} \pi \pi$ with a width of $60 \pm 30 \mathrm{MeV}$. In addition, analogous to the $Y(4660)$, we also predict decays into $\eta_{c}^{\prime} K^{+} K^{-}$and $\eta_{c}^{\prime} \gamma \gamma$. There is also the possibility of a decay into $\Lambda_{c}^{+} \Lambda_{c}^{-}$. We predict the $\pi^{+} \pi^{-}$invariant mass spectrum of the $Y_{\eta}(4616)$ decay into $\eta_{c}^{\prime} \pi^{+} \pi^{-}$, and the prediction is parameter free. We also predict the line shape of the state in the $\eta_{c}^{\prime} \pi^{+} \pi^{-}$final state assuming its decays are saturated by the $\eta_{c}^{\prime} \pi \pi$.

The state can be searched for in the Belle and BABAR $B$ factories. The branching fraction of the $B^{ \pm} \rightarrow$ $\eta_{c}^{\prime} K^{ \pm} \pi^{+} \pi^{-}$is estimated to be of order $1 \times 10^{-3}$, and 
hence there is a great opportunity to find the $\eta_{c}^{\prime} f_{0}(980)$ bound state proposed here in the $\eta_{c}^{\prime} \pi^{+} \pi^{-}$final state of this decay. The state can also be studied with PANDA at FAIR [30] in the future. Such a study would be helpful to understand better not only the $X Y Z$ states observed in recent years but also the interaction between a charmonium and light hadrons, which can provide useful information for understanding the charm production in relativistic heavyion collisions.

This work is partially supported by the Helmholtz Association through funds provided to the virtual institute "Spin and strong QCD" (VH-VI-231) and by the DFG (SFB/TR 16, "Subnuclear Structure of Matter"). We also acknowledge the support of the European CommunityResearch Infrastructure Integrating Activity "Study of Strongly Interacting Matter" (acronym HadronPhysics2, Grant Agreement No. 227431) under the Seventh Framework Programme of EU.

*f.k.guo@fz-juelich.de

†c.hanhart@fz-juelich.de

*meissner@itkp.uni-bonn.de

[1] M. Neubert, Phys. Rep. 245, 259 (1994).

[2] A. V. Manohar and M. B. Wise, Heavy Quark Physics (Cambridge University Press, Cambridge, U.K., 2000).

[3] E. S. Swanson, Phys. Rep. 429, 243 (2006).

[4] E. Klempt and A. Zaitsev, Phys. Rep. 454, 1 (2007).

[5] M. B. Voloshin, Prog. Part. Nucl. Phys. 61, 455 (2008).

[6] S. Godfrey and S. L. Olsen, Annu. Rev. Nucl. Part. Sci. 58, 51 (2008).

[7] T. Barnes, F. E. Close, and H. J. Lipkin, Phys. Rev. D 68, 054006 (2003).

[8] E. van Beveren and G. Rupp, Phys. Rev. Lett. 91, 012003 (2003).

[9] E. E. Kolomeitsev and M. F. M. Lutz, Phys. Lett. B 582, 39 (2004).
[10] F. K. Guo, P. N. Shen, H. C. Chiang, and R. G. Ping, Phys. Lett. B 641, 278 (2006).

[11] F. K. Guo, P. N. Shen, and H. C. Chiang, Phys. Lett. B 647, 133 (2007).

[12] D. Gamermann, E. Oset, D. Strottman, and M. J. Vicente Vacas, Phys. Rev. D 76, 074016 (2007).

[13] A. Faessler, T. Gutsche, V. E. Lyubovitskij, and Y. L. Ma, Phys. Rev. D 76, 014005 (2007).

[14] M.F. M. Lutz and M. Soyeur, Nucl. Phys. A813, 14 (2008).

[15] F. K. Guo, C. Hanhart, S. Krewald, and U.-G. Meißner, Phys. Lett. B 666, 251 (2008).

[16] F. K. Guo, C. Hanhart, and U.-G. Meißner, Eur. Phys. J. A 40, 171 (2009).

[17] C. Amsler et al. (Particle Data Group), Phys. Lett. B 667, 1 (2008).

[18] K. Gottfried, Phys. Rev. Lett. 40, 598 (1978).

[19] M. B. Voloshin, Nucl. Phys. B154, 365 (1979).

[20] Y. P. Kuang, Front. Phys. China 1, 19 (2006).

[21] M. B. Voloshin, Phys. Rev. D 74, 054022 (2006).

[22] S. Dubynskiy and M. B. Voloshin, Phys. Lett. B 666, 344 (2008).

[23] X. L. Wang et al. (Belle Collaboration), Phys. Rev. Lett. 99, 142002 (2007).

[24] An additional momentum factor had to be introduced in this analysis to account for the apparent asymmetry in the spectral function. This asymmetry emerges naturally in the analysis of Ref. [25].

[25] F. K. Guo, C. Hanhart, and U.-G. Meißner, Phys. Lett. B 665, 26 (2008).

[26] S. Weinberg, Phys. Rev. 130, 776 (1963); 131, 440 (1963); 137, B672 (1965).

[27] V. Baru, J. Haidenbauer, C. Hanhart, Yu. Kalashnikova, and A. E. Kudryavtsev, Phys. Lett. B 586, 53 (2004).

[28] N. N. Achasov and A. V. Kiselev, Phys. Rev. D 73, 054029 (2006); 74, 059902(E) (2006).

[29] F. Ambrosino et al. (KLOE Collaboration), Eur. Phys. J. C 49, 473 (2007).

[30] M. F. M. Lutz, B. Pire, O. Scholten, and R. Timmermans (PANDA Collaboration), arXiv:0903.3905. 